\title{
Pixels per Inch
}

National Cancer Institute

\section{Source}

National Cancer Institute. Pixels per Inch. NCI Thesaurus. Code C114239.

A unit of image resolution expressed in the numbers of pixels per inch in the horizontal or vertical direction. 